\title{
Induction of Internal Capacitance Effect in Performance Measurement of OPV (Organic Photovoltaic) Device by RTOSM (Real-Time One-Sweep Method)
}

\author{
Yean-San Long, Shu-Tsung Hsu and Teng-Chun Wu \\ Center for Measurement Standards, Industrial Technology Research Institute, Hsin-Chu 30011, Taiwan
}

Received: February 14, 2014 / Accepted: March 11, 2014 / Published: June 30, 2014.

\begin{abstract}
The advantages of OPV (organic photovoltaic) are low cost, little pollution and flexible. But challenge for OPV manufacture still is lacking of accurately performance measurement due to capacitance issue. Firstly, characterization of OPV requires considering the slowly temporal response due to capacitance effect, and the relative I-V (current-voltage) curves are strongly dependent on the voltage sweep direction, even for the sweep time only in few seconds or less. Secondly, the IPCE (incident photon-to-electron conversion efficiency) also shows the slowly temporal response due to capacitance effect and is dependent on the wavelength of the incident light. Furthermore, the related features for measuring I-V curves are more sensitive with temperature due to non-linear characteristics issue, but current IPCE spectra of OPV are similar to that happened in conventional crystalline Si or amorphous silicon devices. In this work, we developed a RTOSM (real-time one-sweep method) applied both in I-V and IPCE to analysis different electronic transport materials, and result showed this new approach proposed a good way to slow down testing time and having better accuracy for OPV measurement by eliminating acceptance effect instantly.
\end{abstract}

Key words: Organic photovoltaic, real-time removing capacitance effect, one-sweep method.

\section{Introduction}

The DSC (dye-sensitized solar cell) and OSCs (organic polymer solar cells) have been the targets of active research as candidates for next generation OPV (organic photovoltaic) devices. The present study investigates the issues required for the precise characterization of the performance of OPV. Firstly, the following technologies are necessary for the precision measurements of every kind of PV devices: maintaining and confirming the equipment such as the solar simulator, I-V (current-voltage) curve and IPCE (incident photon-to-electron conversion efficiency), etc.. Especially, the irradiance, uniformity, spectrum, and stability of the solar simulator should be carefully checked. Adjusting the irradiance of the solar

Corresponding author: Yean-San Long, researcher, research fields: materials chemistry, instrument analysis and metrology in solar cell. E-mail: mickeylong88@itri.org.tw. simulator by the reference cell, which has the same spectral response as the sample. The spectral responses of the reference cell should be chosen to minimize the spectral mismatch error. In addition to the above basic techniques, understanding the device-dependent features such as the spectral response, linearity for bias light, stability, number of junctions and temporal response, etc. are important for characterizing their performance. It has been known that the performance measurement of the c-Si devices is very dependent on the sweep speed and direction of the I-V measurement, when the sweep speed is shorter than a certain threshold. The mechanism has been discussed related to the capacitance of those devices [1-3]. Typical threshold sweep speed was in the order of millisecond or less, for c-Si devices on the market of early 1990's. Recent organic and high-efficiency solar cells are expected to require much slower sweep 


\section{Induction of Internal Capacitance Effect in Performance Measurement of OPV (Organic Photovoltaic) Device by RTOSM (Real-Time One-Sweep Method)}

speed. Although the absolute value of the peak power (Pmax) or the conversion efficiency is essential in those new devices, the quantitative effect of the sweep speed and direction for those devices is not well established.

We discuss the measurement and analysis of I-V and IPCE characteristics of OPV and discuss both the error sources in the measurements and the strategies to minimize their influence. A brief discussion of the history of photovoltaic efficiency measurements and procedures will be presented. These error sources include the sample area, spectral errors, temperature fluctuations, response time of I-V and IPCE, contacting, and degradation during testing. Information that can be extracted from measurement include Pmax, open-circuit voltage (Voc), short-circuit current (Isc), series and shunt resistance (Rs and Rsh), Fill-factor (FF.\%), dark current, and photo-current. Furthermore, developed a RTOSM (real-time one-sweep method) applied both in I-V and IPCE to analysis different electronic transport materials, and result showed this new approach proposed a better way to saving testing time and having better accuracy for OPV measurement by eliminating acceptance effect instantly.

\section{Experiments}

\subsection{Samples}

Samples were arranged from Taiwanese companies, and major components need include Platinum-coated conducting glass, $\mathrm{TiO}_{2}$-dye electrode and counter electrode. The compositions of electrolyte solution are liquid and ionic.

\subsection{Set-up Experiment Equipment for $O P V$}

OPV, current best photoelectric conversion efficiency, although above to $11 \%$, but its packaged as sandwich structure. However, in recent years, new materials, solar cells and modules have been newly designed device structures toward composite design, the material or component structure no longer belong single material, but also extends the problem in measuring efficiency. Compared with the general type of element except $\mathrm{Si}$ solar cell photo-current nonlinearities, but also for nonlinear temperature changes, such as Fig. $1 \mathrm{c}-\mathrm{Si}$ and the temperature trends OPV schematic diagram to learn from the incident light spectrum and in determining the irradiance, solar cell output performance of its operating temperature have a significant impact. c-Si with an efficiency increase linearly with temperature decrease, while OPV efficiency is indeed nonlinear changes. Similar too many other conventional PV devices, the Voc and Pmax decreased as the temperature increased. The temperature coefficient of Voc (about $-0.1 \% /{ }^{\circ} \mathrm{C}$ ) of the present sample is very small compared to typical c-Si (around $-0.3 \% /{ }^{\circ} \mathrm{C}$ to $-0.5 \% /{ }^{\circ} \mathrm{C}$ ). The temperature coefficient of Isc (slightly negative) is also different from many other $\mathrm{c}-\mathrm{Si}$ devices (usually around $+0.05 \% /{ }^{\circ} \mathrm{C}$ to $+0.1 \% /{ }^{\circ} \mathrm{C}$ ), although the detail of the Isc variation will require further confirmation.

Also, investigation on the variation of the quantum efficiency and spectral mismatch correction will be important. It is again noted that although these temperature-dependence and irradiance dependence were similar among the samples of the present study, they can be strongly dependent on the device design.

A longer illumination time is critical to test new material of solar cell devices, since the response time is significantly longer than the regular flash testing method of 10 milliseconds to 100 milliseconds. The

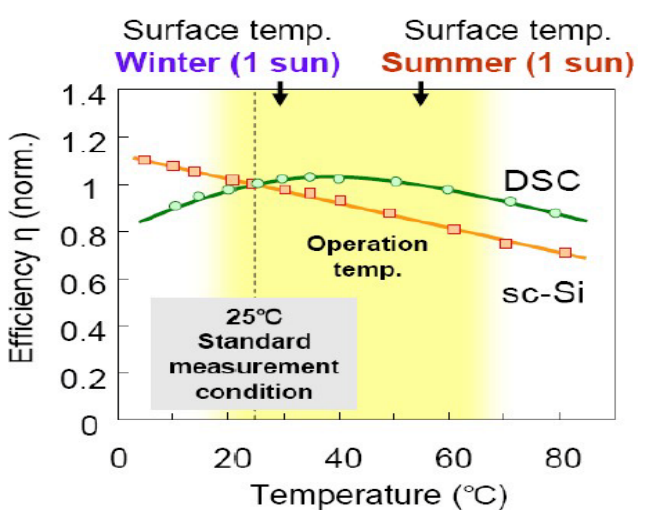

Fig. 1 c-Si and OPV temperature fluctuations [4]. 
response time of the new devices is affected by: (1) capacitive effects at large module sizes and powers, Ex. CIGS/Amorphous silicon/...; (2) chemical kinetics, Ex. OPV/OSC/DSC/...; (3) module integrated electronics. Efficiency due to capacitance effects caused by measurement errors, such as Fig. 2-I-V measured capacitance effects generated diagram can be understood from figure cause measurement Pmax, Voc and Isc of the error, the current solution methods Hold-Time single points, Multi-flash single points and so on.

Generally, the performance of solar cells is determined by analyzing I-V curves measured under simulated AM $1.5 \mathrm{G}$ sun light. A high quality solar simulator is thus required for indoor measurement. The most common type of light source for solar simulators is the Xenon lamp that can provide illumination for the measurement of different sample areas with various light intensities and the simulated spectrum can nearly match the spectrum of sunlight from the visible to near IR range. However, there are some unfavorable sharp atomic transitional peaks around $450 \mathrm{~nm}$ and $650 \sim 850$ $\mathrm{nm}$ in the spectrum of the commercial solar simulator. These peaks give high intensity light that can induce extra photocurrent in the evaluation of OPV. For example, the spectral response of a DSC with the N719 dye mainly covers the wavelength range of $300 \mathrm{~nm}$ to $750 \mathrm{~nm}$ and errors can be caused by the extra light peaks around 450 700 $\mathrm{nm}$. In addition, the spectrum and light intensity of the lamp varies with its aging process. Therefore, it is often required to calibrate the solar simulator to minimize the mismatch of the spectra. Since the reference cell is important in the calibration of the solar simulator, it is required long-term stability for frequent calibrations. Until now, a DSC matching such requirements has rarely been found. Therefore, a c-Si reference cell with some filters which is long-term stability is generally used as an alternative. Table 1 shows as for instance, a KG-5 filter used during calibration would allow the reference cell to have a similar spectral response to a DSC with N719 dye. In

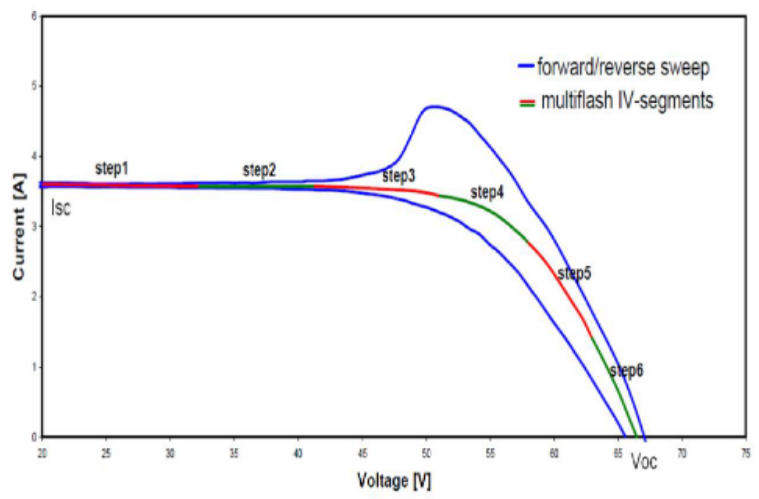

Fig. 2 I-V measurements diagram capacitive effect produced [5].

Table 1 The filtered c-Si reference cell.

\begin{tabular}{ll}
\hline Windows with FilterType & Applied type \\
\hline $\mathrm{Si}+\mathrm{BK} 7$ or Quartz & Mono-crystalline Si (c-Si) \\
$\mathrm{Si}+\mathrm{BK} 7$ or Quartz & Poly-crystalline Si (mc-Si) \\
$\mathrm{Si}+\mathrm{BK} 7$ or Quartz with KG2 & Amorphous $\mathrm{Si}(\mathrm{a}-\mathrm{Si})$ \\
$\mathrm{Si}+\mathrm{BK} 7$ or Quartz with KG3 & $\begin{array}{l}\text { DSC with N749 } \\
\text { DSC with N719 and OPV } \\
\mathrm{Si}+\mathrm{BK} 7 \text { or Quartz with KG5 with polymer BHJ (Bulk } \\
\text { heterojunction) }\end{array}$ \\
\hline
\end{tabular}

addition, when test DSC with N749 dye, the solar simulator needs to be calibrated with a c-Si reference cell covered by the KG-3 filter. For DSC with other dyes, such as newly developed organic dyes with IPCE spectra in the range of $300 \mathrm{~nm}$ to $700 \mathrm{~nm}$, narrower than that of N719, theoretically new corresponding reference cells are required for every specific test DSC. However, it is very hard to realize such strict conditions in practice. An effective solution is to use the existing reference cell of DSC sensitized with N719 for calibration because it can avoid the errors induced by the peaks over $750 \mathrm{~nm}$. In the near future, if we could develop OPV with an IPCE extending further into infrared region, a careful selection of other suitable filters will be needed to adjust the IPCE spectra of the reference solar cells to match that of the test OPV in the calibration. The use of a light adjustment filter during calibration of the solar simulator is a necessary technique to ensure that the input light for testing is suitable for the tested solar cell, and thus reduce measurement errors $[1,2,4]$.

Because of multiple sources of error will cause the 
efficiency of the overall measurement error, so we integrate existing measurement device characteristics related functions, such as the integrated $\mathrm{I}-\mathrm{V}$ measurement wasperformed using a digital source meter in conditions of illumination (AM 1.5 G, 100 $\mathrm{mW} / \mathrm{cm}^{2} ; \quad$ IPCE (PV Measurements, Inc.) measurement was performed using PV measurement with theisothermaltest platform, and with the software by home-made immediately removed due to capacitance effects caused efficiency measurement errors, and to achieve a measure reducing the measurement result due to degradation.

\section{Test and Analysis}

\subsection{Testing Flow Chart}

The measured performance of OPV is highly responsive to the external measurement conditions. A reliable evaluation report requires the measurement to be performed under proper conditions and the details of the measurement should be clearly described in the report. A list of proposals for the measurement of $\mathrm{OPV}$ is given below:

\section{- Visual inspection}

For the purposes of design qualification and type approval, the following are considered to be major defects, Ex. Bubbles or delamination forming a continuous path between any part of the electrical circuit and the edge of the DSC, etc..

- I-V and IPCE measurement system

An AM $1.5 \mathrm{G}$ solar light simulator should be calibrated by a reference cell, generally a filtered c-Si solar cell (show as Table 2), which has a similar IPCE spectrum compared to the test OPV. Then PV reference device shall either be spectrally matched to test specimen, or a spectral mismatch correction shall be performed in conformance with IEC 60904-7.

The temperature of reference device and the specimen shall be measured by temperature recorder with the accuracy of $\pm 1{ }^{\circ} \mathrm{C}$ and repeatability of $\pm 0.5^{\circ} \mathrm{C}$. If the temperature of reference device differs and more than $2{ }^{\circ} \mathrm{C}$ from the temperature at which it
Table 2 Remove the capacitance effect in Forward/Backward curve difference comparison table.

\begin{tabular}{llll}
\hline Liquid-Type & & & \\
\hline Item & Pmax & Isc & Voc \\
\hline Forward & 1.4500 & 2.5248 & 0.7693 \\
Backward & 1.4611 & 2.5382 & 0.7709 \\
F-B & 0.0111 & 0.0134 & 0.0016 \\
F/B & 0.9924 & 0.9947 & 0.9979 \\
\hline Ionic-Type & & & \\
\hline Item & Pmax & Isc & Voc \\
\hline Forward & 0.003597 & 0.009473 & 0.650537 \\
Backward & 0.003604 & 0.009567 & 0.648149 \\
F-B & 0.0000070 & 0.0000940 & -0.0023880 \\
F/B & 1.0019 & 1.0099 & 0.9963 \\
\hline
\end{tabular}

was calibrated, the calibration value shall be adjusted to the measured temperature. If the reference device is a pyranometer, then temperature measurement and temperature correction are not required for output signal. During the measurement, the OPV temperature should be stable at $25^{\circ} \mathrm{C}$ with a fluctuation of less than 1 degree. Illuminating test OPV for a long time before starting measurement is recommended to stabilize the heat generated by light absorption in the cell.

- Electrical signal connecting

Voltages and currents shall be measured by using source meter with an accuracy of $\pm 0.2 \%$ of the Voc and Isc, and using independent leads from the terminals of the specimen to keep them as short as possible. The measurement ranges of the data acquisition should be carefully chosen. If the test specimen is a packaged OPV, the two (or four) terminal wires connection should start at the cell bus bars. Connect checking by the Isc shall be measured, using a variable bias (preferably electronic) to offset the voltage drop across the external series resistance. Alternatively, Isc may be extrapolated from the I-V characteristic. The I-V curve is extrapolated to zero voltage provided that voltage drop is not higher than 3\% of the device Voc and that there is a linear relationship between current and voltage.

- Sample precondition time

To annealing stabilize the electrical characteristics of OPV by means of simulated solar irradiation approach $10 \mathrm{~min}$ to $30 \mathrm{~min}$, some requirement is 
written as follows:

(1) Using the reference device, set the irradiance between $800 \mathrm{~W} / \mathrm{m}^{2}$ and $1,000 \mathrm{~W} / \mathrm{m}^{2}$, and record the irradiance;

(2) Set OPV at Bias $=-0.05 \mathrm{~V}$ and mount them, recommend operated by the manufacturer in the test plane of the simulator;

(3) Ensure OPV temperature does not vary by more than $\pm 2{ }^{\circ} \mathrm{C}$ within the range of $40 \sim 50{ }^{\circ} \mathrm{C}$ during the test. Record the temperature.

- Forward and backward

We read simultaneously multi-point forming step after taking the optimization stabilizing area as a point on the I-V curve. That I-V measurements of both scan directions are necessary to estimate the related measurement error, as the temporal response is expected to be dependent on the device structure of OPV and the I-V curve measured at the different sweep directions tend, although the difference very closed in Pmax/Voc/Isc less than $0.2 \% \sim 0.5 \%$.

\section{- Light I-V at STC}

Each sweep direction (Forward or backward) need test at least five times. And the RSD (relative standard deviation) is expressed in percent, obtained by multiplying 100 and dividing this product by the average. That RSD of Pmax/Voc/Isc measured at each sweep direction should be less than $1.5 \%$. Then non-uniformity measured from Pmax/Voc/Isc due to each sweep direction is defined as $(\max -\min ) /(\max$ $+\min ) \times 100 \%$, where the maximum and minimum are the values measured of Pmax/Voc/Isc due to each sweep direction, and all should be less than $1.5 \%$.

- Data analysis

(1) I-V characteristic parameter: The Calculation method of Voc, Isc, Pmax, FF, effectively can be applied using either ASTM E948 or US PTO. 8224598 [6].

(2) Calibration of OPV against a reference cell for mismatch correction method is used by IEC 60904-7.

\subsection{I-V Characteristics}

We follow testing flow chart (Fig. 3) in the I-V

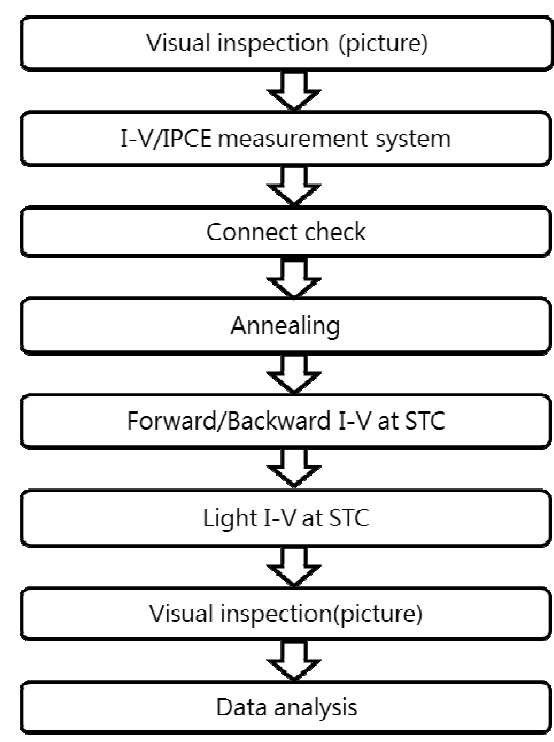

Fig. 3 Testing flow-hart.

measurement, the applied bias voltage is changed stepwise from Isc to Voc (forward scan) or in the reverse direction (backward scan, Voc to Isc). When there is capacitance effect occurs, this measurement process will produce as Fig. 4 of the phenomenon. The time constant for equilibrium of OPV can be estimated by the measurement of the transient photocurrent under the application of a stepwise-changed voltage. Fig. 4 shows the stepwise applied voltage around Voc in a step of $10 \mathrm{mV}$ with 1 ms sampling time and the transient photocurrent responses of the DSC both in forward and backward scans. In the forward scan, an overshot current appears immediately after an abrupt increase of applied voltage and then the current gradually decreases to an equilibrium state. So this figure I-V measurements for the capacitive effects generated during real-time monitoring chart, graph we can take advantage of this monitoring did not produce capacitive effect immediately find a flat area, take advantage of this flat area can be re-drawn a positive sweep and reverse sweep I-V curve by curve available as Fig. 5 and Table 2 remove the capacitance effect in Forward/backward curve difference comparison table. From Fig. 5 and Table 1, we can already understand this immediately remove the capacitive effect of the average method to get the complete removal of 

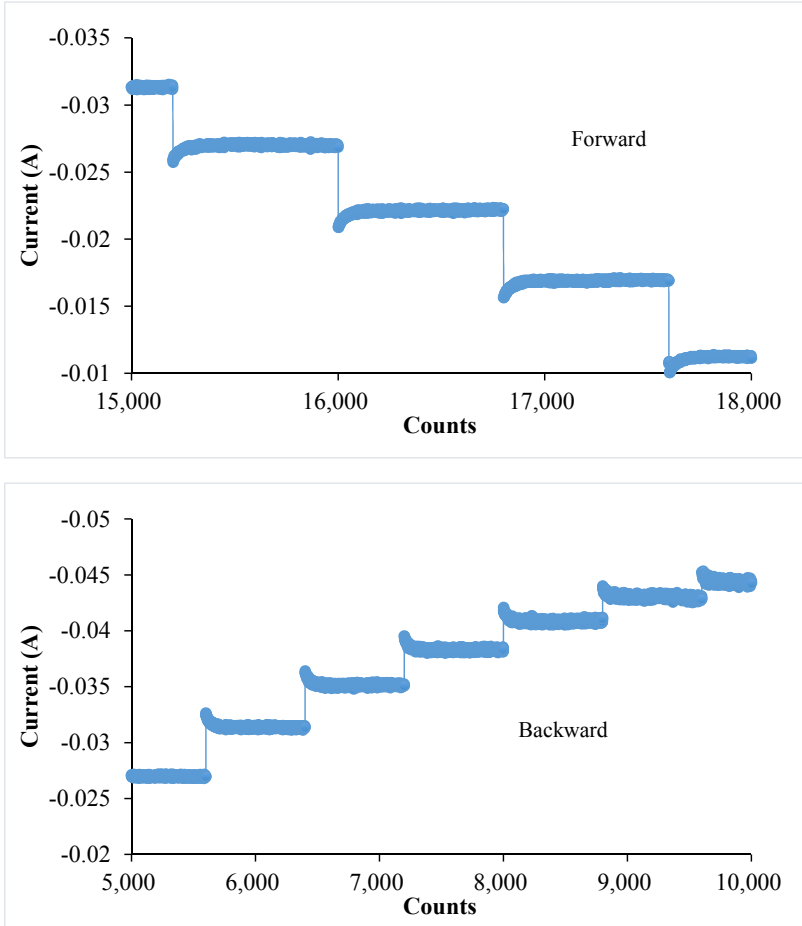

Fig. 4 I-V measurement occurs capacitance effect.

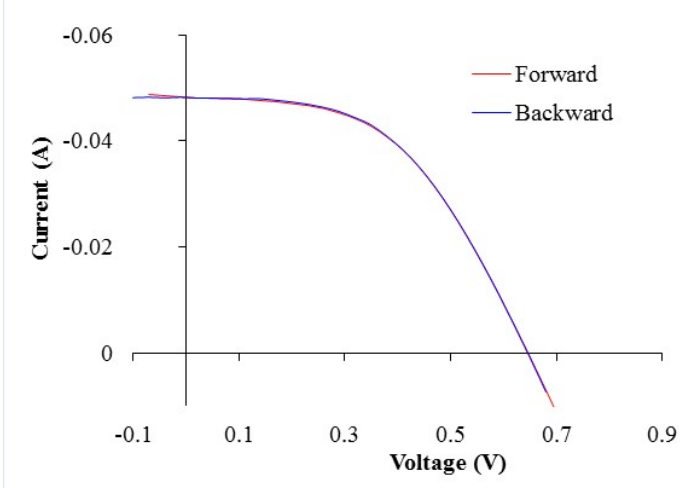

Fig. 5 I-V measurement in forward (red)/backward (blue) curve.

capacitance effects. Therefore, the temporal stability of the output current should be measured at a fixed bias voltage as shown in Fig. 1 and Table 2, in order to confirm the "true" Pmax of OPV, especially for samples which have strong dependence on the sweep direction.

\subsection{IPCE Characteristics}

The spectral response was investigated by the AC and DC modes at different bias light levels.

When the bias light is not applied, the quantum efficiency spectra measured by the AC mode showed strong dependence on the chopping frequency of the monochromatic light between 1.1-3.4 Hz. AC and DC results agreed under a bias light of $100 \mathrm{~mW} / \mathrm{cm}^{2}$, although the drift of the DC current prevented precise comparison of the $\mathrm{AC}$ and $\mathrm{DC}$ measurements. When the bias light is not applied for the AC mode, on the other hand, the measured result is strongly dependent on the frequency, especially at longer wavelength. With this real-time average method removes capacitance effects obtained under Isc in IPCE if the results of Fig. 6, shows as Isc IPCE and I-V in the comparison chart, and linear regression analysis, the results of $R^{2}=0.999939$, we can see very close, which means that the IPCE Isc under actual measured under the I-V Isc very consistent.

This indicates the IPCE spectra measured in the DC mode could be reliable if the measurement conditions are properly considered. The DC mode would only be reliable when Isc has a linear relationship as a function of light intensity.

In the case of OPV with a non-linear relationship, such as DSC based on ionic liquids, the DC mode is suitable anymore. Therefore, we recommend that the IPCE spectrum should be measured in the DC mode or the $\mathrm{AC}$ mode at a frequency low enough for the illumination of simulated solar light.

\section{Discussion}

In this study, we used real-time removing Capacity Effect (RTOSM) in I-V and IPCE measurement [7]. It

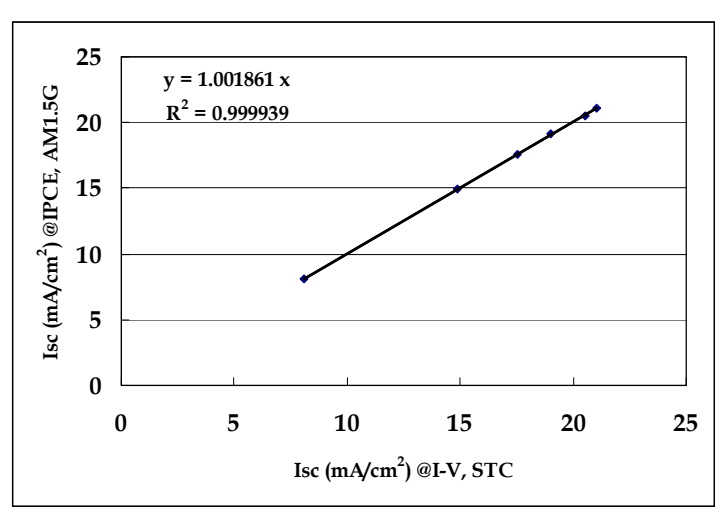

Fig. 6 Isc in IPCE compared with the I-V. 
has been investigated that we get performance characterization of the OPV. Firstly, optical and electrical considerations such as adjusting the solar simulators are common to the performance measurements of any type solar cells including the OPV. Accurate then gradually decreased (Round dot), so call "Capacity Effect". We now consider appropriate conditions of I-V measurements for DSCs. True cell performance must be measured under conditions of equilibrium, in which the photocurrent is stable (Square dot). To satisfy these conditions, we read simultaneously multi-point forming step after taking the optimization stabilizing area as a point on the I-V curve. Characterization of the I-V curves and quantum efficiency of DSC further requires consideration on the very slow temporal response of the order of seconds or minutes, and the effect of bias light intensity. Fig. 7 shows the transient photocurrent of the DSC in the case of a forward scan, that is, stepwise voltage is positively applied. It was found that an overshot current could be observed immediately after an abrupt increase of voltage; although the temporal and spectral characteristics are dependent on the device design, the present results can be utilized for precisely characterizing the I-V curves and spectral responses of the DSC. From this study aims to address the emerging photovoltaic products with existing measurement errors, the current on the other hand, the characterization-related features for measuring the I-V curves and quantum efficiency spectra of OPV are rather similar to conventional crystalline $\mathrm{Si}$ or amorphous silicon devices. the analysis methods and the existing removal efficiency measurement error method is as Table 3, from tables to learn about our approach to real-time completely removed, as Table 2 and from Fig. 4 have reached the proper effect.

\section{Conclusions}

However, an appropriate tracing and measurement both voltage and current simultaneously are useful in detecting problems from capacitance of solar cell. Generally, the real-time measurement shall have a shift in the shape of I-V curve, and led to output the wrong maximum power of solar cell. The decay of photocurrent exposed the worse condition of capacitance while peak power voltage is higher. In this work, RTOSM can remove the capacity effect in real-time for I-V and IPCE successfully. Result also demonstrated the influence from OPV capacitance during the measurement of I-V curves by using a steady-state simulator. RTOSM [8] certainly speeds up the characteristic research of OPV to get more reasonable result (Table 2, Fig. 5).

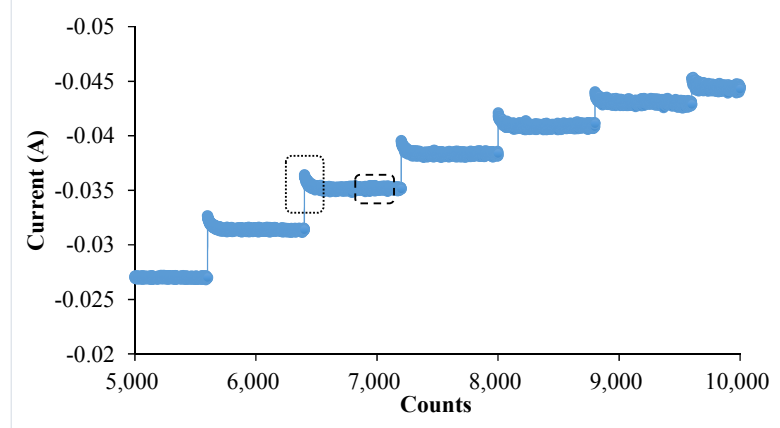

Fig 7 Typical signals obtained in transient photocurrent measurements after application of stepwise voltage with sampling time of $1 \mathrm{~ms}$ for DSC [7].

Table 3 Remove the capacitive effect of the measurement method comparison table.

\begin{tabular}{|l|l|l|l|}
\hline Item & $\begin{array}{l}\text { Dynamic measure-single points } \\
\text { (Hold-time) [8-11] }\end{array}$ & Multi-flash single points [2,3] & One-sweep method [7] \\
\hline Measurement & $\begin{array}{l}\text { Meanwhile, after reading the multi-point } \\
\text { forming step, take a little at steady state } \\
\text { as a point on the I-V curve }\end{array}$ & $\begin{array}{l}\text { While the solar simulator for } \\
\text { multiple flashes, take the point } \\
\text { as a point on the I-V curve }\end{array}$ & $\begin{array}{l}\text { Read simultaneously multi-point forming } \\
\text { step taking the average area at } \\
\text { steady state as a point on the I-V curve }\end{array}$ \\
\hline Results & $\begin{array}{l}\text { Forward/backward sweep the I-V curve is } \\
\text { the same in a certain range }\end{array}$ & $\begin{array}{l}\text { Forward/backward sweep the } \\
\text { I-V curve is the same in a a a } \\
\text { certain range }\end{array}$ & $\begin{array}{l}\text { Forward/Backward is consistent with the } \\
\text { I-V curve sweep, the difference 0.2\% or } \\
\text { less. }\end{array}$ \\
\hline Difference & An analysis of the results obtained & $\begin{array}{l}\text { An analysis of the results } \\
\text { obtained }\end{array}$ & $\begin{array}{l}\text { Once for the complete data and analysis } \\
\text { results }\end{array}$ \\
\hline
\end{tabular}




\section{References}

[1] V. Shrotriya, G. Li, Y. Yao, T. Moriarty, K. Emery, Y.Yang, Accurate measurement and characterization of organic solar Cells, Adv. Funct. Mater 16 (2006) 2016-2023.

[2] H. Ossenbrink, A. Drainer, W. Zaaiman, Errors in current-voltage measurements of photovoltaic devices introduced by flash simulators, in: 10th EUPVSEC, Kluwer, 1991.

[3] S. Mau, Influence of solar cell capacitance on the measurement of I-V curves of PV-Modules, in: Proc. 20th EUPVSEC, Barcelona, Spain, 2005.

[4] M.A. Green, K. Emery, Y. Hishikawa, W. Warta, E.D. Dunlop, Solar cell efficiency tables (version 39), Prog. Photovolt: Res. 20 (1) (2012) 12-20.

[5] D. Willett, S. Kuriyagawa, The effects of sweep rate, voltage bias and light soaking on the measurement of CIS-based solar cell characteristics, in: Proc. 23rd IEEE PVSEC, Berlin, 1993, pp. 495-499.
[6] Method for forming optimal characteristic curves of solar cell and system thereof, US PTO 8224598, 2012.

[7] T.C. Wu, S.T. Hsu, Y.S. Long, New set-up procedures and integrated measurement system for OPV (organic photovoltaic) module, PVSEC 23 (2013) 1024-1026.

[8] W.M. Keogh, A.W. Blakers, A. Cuevas, Constant voltage I-V curve flash tester for solar cells, Solar Energy Materials and Solar Cells 81 (2004) 183-196.

[9] S. Ito, H. Matsui, K. Okada, S. Kusano, T. Kitamura, Y. Wada, et al., Sol. Energy Mater. Sol. Cells 82 (2004) 421-429.

[10] A. Cuevas, F. Recart, Capacitive effects in quasi-steady-state voltage and lifetime measurements of silicon devices, Journal of Applied Physics 98 (7) (2005) 1-7.

[11] H. Kathleen, F. Ian, M. Robert, N. Paresh, L. David, R. Keith, et al., The manufacture of CIGS based thin film PV cells with minimal environmental impact, in: 2nd Photovoltaic Science, Applications and Technology Conference, 2005, pp. 11-15. 